\title{
Analysis of IL-6, IL-1 $\beta$ and TNF- $\alpha$ production in monocytes isolated from multiple sclerosis patients treated with disease modifying drugs
}

\author{
Sarah E Fiedler ${ }^{1}$, Joshua D George ${ }^{1}$, Haley N Love ${ }^{1}$, Edward Kim ${ }^{1,2}$, Rebecca Spain ${ }^{1,2}$, Dennis Bourdette ${ }^{1,2}$ and Sonemany Salinthone $^{1,2 *}$ \\ ${ }^{1}$ VA Portland Health Care System, Research and Development Service, 3710 SW US Veterans' Hospital Rd. Portland, OR 97239, USA \\ ${ }^{2}$ Department of Neurology, Oregon Health \& Sciences University, 3181 SW Sam Jackson Park Rd., Portland, OR 97239, USA
}

\begin{abstract}
Objective and design: The etiology of multiple sclerosis (MS) is unknown, but blood derived monocytes/macrophages are believed to be involved in the pathogenesis through phagocytosis of myelin and production of inflammatory mediators. The objective of this study is to examine inflammatory cytokines that are present at elevated levels in active MS lesions to determine whether there are differences between classically stimulated monocytes isolated from healthy control (HC) and relapsing-remitting MS (RRMS) subjects taking disease modifying drugs (DMDs), including dimethyl fumarate (DMF).
\end{abstract}

Subjects: Thirty-nine veterans of the US Armed Forces were enrolled, 21 health controls (HC), and 18 with relapsing-remitting MS (RRMS), all taking DMDs.

Methods: Use ELISAs to measure production of IL-6, IL-1 $\beta$ and TNF- $\alpha$ by LPS-stimulated peripheral monocytes.

Results: Activation of monocytes from MS subjects produced significantly more IL-6 than healthy controls (49531 \pm 20795 vs. 10526 \pm 4845$)$, and IL-6 production trended higher in MS subjects taking DMF than those taking other DMDs $\quad(72186.9 \pm 35156.2$ vs. $32585.8 \pm 17135.4)$. There were no significant differences in IL-1 $\beta$ or TNF- $\alpha$ secretion.

Conclusions: Our data suggest that not all DMDs may provide disease modification by suppressing monocyte/macrophage production of pro-inflammatory mediators.

\section{Introduction}

Multiple sclerosis (MS) is a disease of the central nervous system (CNS) characterized by infiltration of inflammatory cells into the CNS and eventual neurodegeneration due to axonal and oligodendrocyte loss. MS is a T-cell mediated disease, but other immune cell types, including B-cells and peripheral monocytes which migrate into the CNS to become macrophages, contribute to disease pathogenesis. Bloodderived monocytes and CNS macrophage-related microglial cells are believed to be essential in both the initial and sustained inflammatory response to myelin antigens in MS. Depletion of macrophages in acute experimental autoimmune encephalitis (EAE), a rodent model of MS, results in suppression of clinical signs and a reduction of infiltrated macrophages in the CNS [1-3]. Macrophages are the major responders to CNS chemokines, produce toxic and pro-inflammatory molecules that promote demyelination, and are the major cell type involved in phagocytosis/degradation of myelin sheathes [4]. Active MS lesions contain macrophages which are believed to contribute to lesion formation by various mechanisms $[5,6]$.

There are currently no treatments that can halt or reverse MS progression, likely due to the complex nature of disease pathogenesis and lack of knowledge on disease etiology. A number of disease modifying drugs (DMDs) have been developed that can alter disease course - reducing relapse rates and slowing disability progression. These DMDs include interferons, natalizumab, fingolimod, rituximab, ocrelizumab and dimethyl fumarate (DMF), the most recently approved oral drug for treatment of the relapsing phase of MS [7]. In vitro studies have shown that DMF decreases synthesis of the proinflammatory mediators TNF- $\alpha$, IL- $1 \beta$ and IL- 6 at the RNA level in activated microglia and astrocytes [8], inhibits TNF- $\alpha$, IL-12 and IFN- $\gamma$ secretion in LPS-activated healthy human PBMCs, and reduces IL-6, TNF- $\alpha$, NO, and ROS production in macrophages $[9,10]$. Despite this increasing body of in vitro data, less is known concerning the effects of DMF on the inflammatory cytokine response of peripheral immune cells isolated from patients under treatment (ex vivo response), specifically the effects on monocytes/macrophages leaving gaps in understanding its mechanisms of action.

In this study, we examined a set of inflammatory cytokines that are present at elevated levels in active MS lesions (IL-1 $\beta$, TNF- $\alpha$, and IL-6) [11-13] and sought to determine whether there were differences in production of these cytokines in classically stimulated monocytes isolated from relapsing remitting MS (RRMS) subjects taking DMF compared to subjects that are on other DMDs and healthy controls (HC).

Correspondence to: Sonemany Salinthone, VA Portland Health Care System, Mail Code R\&D8, 3710 SW US Veterans Hospital Road, Portland, OR 97239, USA, Tel: 503-220-8262 extension 54394; Fax: 503-402-2816; E-mail: salintho@ohsu.edu

Key words: multiple sclerosis, dimethyl fumarate, monocyte, macrophage, IL-6 Received: May 28, 2017; Accepted: June 15, 2017; Published: June 17, 2017 


\section{Materials and methods}

\section{Subjects}

This study received approval from the VA Portland Healthcare System (VAPORHCS, IRB \#2993) and Oregon Health \& Science University (OHSU) Institutional Review Boards (IRB \#8908) prior to initiation. HC and RRMS subjects were recruited at VAPORHCS via flyers, VAPORHCS MS clinic, and word of mouth. Interested subjects were phone-screened for eligibility prior to scheduling an enrollment visit. To qualify, HC subjects needed to be an adult veteran of the US Armed Forces at least 18 years of age able to provide informed consent, and meet none of the following exclusion criteria: 1) self-reported current substance abuse (except tobacco), alcohol abuse/dependence or sobriety for less than 90 days, 2) pregnant or breast-feeding, 3) anemia as indicated by POC hemoglobin $<12,4$ ) history of bleeding disorders, 5) current anti-coagulant use such as Heparin, Coumadin, or daily aspirin, 6) weight less than $110 \mathrm{lbs}$., 7) other significant inflammatory diseases or health problems (e.g. active coronary heart disease, diabetes mellitus, other auto-immune disorder), or 8) active infection (as indicated by a fever). In addition to the above inclusion/ exclusion criteria, patients enrolled as MS subjects needed to have a current diagnosis of RRMS (confirmed by a neurologist), and were excluded for experiencing MS exacerbation(s) or receipt of systemically administered corticosteroids within 30 days of the blood draw.

\section{Treatment, specimen collection and processing}

Subjects were asked to abstain from alcohol for $24 \mathrm{~h}$ and fast for $11 \mathrm{~h}$ prior to the scheduled visit. Study appointments began with subject eligibility screening and informed consent. Medical history questionnaire (including current medication and supplement listing) was completed, vital signs were measured and recorded, and pregnancy test (if applicable) and anemia checks were performed. Provided all inclusion/exclusion criteria were met, subjects were enrolled in the study, and approximately $400 \mathrm{ml}$ of blood was drawn. Following blood draw, subjects received their choice of continental breakfast options, completing the appointment. Subjects who expressed interest in maintaining study enrollment were re-contacted at later dates to return for additional blood draws.

Blood was collected in $450 \mathrm{ml}$ capacity Anticoagulant Citrate Dextrose Solution Formula A BLOOD-PACK units (Fenwal, Lake Zurich, IL) and immediately processed. To obtain peripheral blood mononuclear cells (PBMCs), whole blood was diluted approximately 1:1 in PBS containing 2\% fetal bovine serum (FBS, Gibco/Thermo Fisher, Waltham, MA), then centrifuged at $200 \mathrm{x}$ g for $10 \mathrm{~min}$ to remove plasma. PBMCs were isolated using Ficoll gradient separation with SepMate tubes (STEMCELL Technologies, Cambridge, MA) according to manufacturer's instructions. After washing in EasySep buffer (STEMCELL Technologies) and RPMI (Gibco/Thermo Fisher, Waltham, MA), cells were assessed for viability, counted, and resuspended in cryopreservation media (RPMI with 25\% FBS and $12.5 \%$ DMSO). Cells were cryopreserved for future experiments at 20 or 40 million cells in $1.5 \mathrm{ml}$ media/tube.

\section{Monocyte enrichment, cell stimulation and enzyme-linked immunosorbent assays (ELISA)}

Cryopreserved PBMCs were quick thawed in a $37^{\circ} \mathrm{C}$ water bath, diluted in RPMI media and washed 3x. Cells were treated with DNase I (final concentration $0.6 \mathrm{mg} / \mathrm{ml}$, Roche, Indianapolis, IN) for $15 \mathrm{~min}$ at $37^{\circ} \mathrm{C} / 5 \% \mathrm{CO}_{2}$, counted, spun down at $300 \mathrm{x} \mathrm{g}$ for $10 \mathrm{~min}$, then re- suspended in monocyte enrichment media (RPMI + L-glutamine, $1 \mathrm{X}$ Pen/Strep, $5 \mathrm{mM}$ Glucose) and plated in 24 well plates $\left(2 \times 10^{6}\right.$ cells $/$ well $)$. Plates were incubated for $90 \mathrm{~min}$ at $37^{\circ} \mathrm{C} / 5 \% \mathrm{CO}_{2}$ to allow monocytes to adhere. After incubation period, monocyte enrichment media was discarded and wells were washed $3 \mathrm{x}$ with $\mathrm{RPMI}+\mathrm{L}$-glutamine to completely remove non-adherent cells. Wells were visually checked for flat monocytes prior to addition of $500 \mu \mathrm{l}$ of monocyte enrichment media to each well. To classically stimulate monocytes, LPS (Santa Cruz Biotechnology, Santa Cruz, CA) was added to wells at a final concentration of $1 \mu \mathrm{g} / \mathrm{ml}$. Control (untreated, UNT) wells received no LPS. After $15 \mathrm{~min}$, FBS ( $0.1 \%$ final concentration) was added to in each well, and plates were incubated $24 \mathrm{~h}$ at $37^{\circ} \mathrm{C} / 5 \% \mathrm{CO}_{2}$. To prepare samples for ELISA analyses, supernatants were collected on ice and cellular debris was removed by centrifugation. IL- $1 \beta$, TNF- $\alpha$, and IL- 6 Ready-Set-Go! ELISAs (eBioscience/Thermo Fisher, Waltham, MA) were performed in triplicate according to manufacturer's protocols, and plates were read using a SpectraMax M2 microplate reader (Molecular Devices, Sunnyvale, CA).

\section{Data and statistical analyses}

Basal (untreated, UNT) and LPS-stimulated values for each subject were determined as an average of the (3) replicates, which were generated from internal 4 parameter logistic (4pl) standard curves using Softmax software (Molecular Devices). These values were used to determine the arithmetic mean, plus or minus the standard error of the mean, of each treatment by subject group. The changes in cytokine levels were determined by subtracting the unstimulated (basal, UNT) value from the LPS-stimulated value for each subject (determined as above), then calculating the arithmetic mean of each group, plus or minus the standard error of the mean. Subject groups were tested for significant differences in baseline demographics, clinical characteristics and cytokine production in Microsoft Excel using one-way ANOVAs and two-tailed Student's t-tests, with results considered significant at $\mathrm{P} \leq 0.05$.

\section{Results}

\section{Subject profile}

Subject demographics and clinical characteristics are listed in Table 1. There were no significant differences in demographics between groups (age, sex, or race). Post study enrollment, an ancillary component was added to examine the effects of DMF treatment compared to other DMDs. Eight of the 18 RRMS subjects were taking DMF. Seven DMF subjects took the standard oral dose of $240 \mathrm{mg}$ twice a day. The $8^{\text {th }} \mathrm{DMF}$ subject took $240 \mathrm{mg}$ twice a day orally every other day. All 10 of the RRMS subjects not taking DMF were taking a single disease modifying drug (DMD); 1 fingolimod, 3 glatiramer acetate, 3 natalizumab, and 3 IFN- $\beta-1 \mathrm{a}$. There were no significant differences in disease duration or severity (based on EDMUS scale scores) of RRMS subjects who were taking DMF vs those who were not. There was a significant difference in the duration of time subjects had been taking their current DMD between RRMS subjects taking DMF (RRMS+DMF) and those who were on other DMDs (RRMS no DMF); this is a result of the relatively recent approval of DMF (March 2013) compared to all other DMDs.

\section{Inflammatory response of classically activated monocytes in HC and RRMS subjects +/- DMF}

IL-6: Basal (UNT) IL-6 levels did not vary significantly between subject groups. However, the amount of IL-6 produced in response to LPS stimulation was significantly greater in monocytes/macrophages 
Table 1. Subject demographics and clinical characteristics

\begin{tabular}{|c|c|c|c|c|}
\hline & Healthy Control & $\begin{array}{l}\text { Relapsing } \\
\text { remitting MS }\end{array}$ & $\begin{array}{l}\text { RRMS } \\
\text { no DMF }\end{array}$ & $\begin{array}{l}\text { RRMS } \\
+ \text { DMF }\end{array}$ \\
\hline Number in group ${ }^{1}$ & 21 & 18 & 10 & 8 \\
\hline$\%$ Male & 85.7 & 88.9 & 90 & 87.5 \\
\hline \multicolumn{5}{|c|}{ Age (years) } \\
\hline Median & 44 & 40.5 & 45 & 36 \\
\hline Range & $27-68$ & $28-61$ & $30-61$ & $28-58$ \\
\hline \multicolumn{5}{|c|}{ Race } \\
\hline$\%$ Caucasian & 90.5 & 83.3 & 70 & 100 \\
\hline \multicolumn{5}{|c|}{ MS duration (years) } \\
\hline Median & NA & 6 & 6 & 5.5 \\
\hline Range & NA & $1-19$ & $1-19$ & $2-10$ \\
\hline \multicolumn{5}{|c|}{${\mathrm{EGS} / \mathrm{DSS}^{2}}^{2}$} \\
\hline Median & NA & 2 & 2 & 2 \\
\hline Range & NA & $2-6$ & $2-6$ & $2-6$ \\
\hline \multicolumn{5}{|c|}{ Current DMD duration ${ }^{3}$ (years) } \\
\hline Median & NA & 2.5 & $5^{*}$ & $1 *$ \\
\hline Range & NA & $0.25-15$ & $2-15$ & $0.25-2$ \\
\hline
\end{tabular}

${ }^{1}$ Not all subjects' blood samples yielded enough PBMCs to complete all ELISAs; specific $\mathrm{N}$ for each ELISA set is thus given in the figure legends

${ }^{2}$ The EDMUS Grading Scale score

${ }^{3}$ Disease modifying drug

* Difference is significan

from RRMS subjects compared to HC (Figure 1A and Supplemental Table 1, $\mathrm{p}=0.042)$ ). The significance of this difference is driven by RRMS subjects that are taking DMF [Figure 1B, note that the difference between HC and RRMS+DMF is significant $(p=0.017)$, while the difference between HC and RRMS is not].

IL-1 $\beta$ and TNF- $\alpha$ : The amounts of IL- $1 \beta$ and TNF- $\alpha$ produced in response to LPS stimulation did not differ between monocytes/ macrophages from $\mathrm{HC}$ and RRMS subjects (Figures $2 \mathrm{~A}$ and $3 \mathrm{~A}$, respectively). The basal (UNT) levels were also not significantly different (see Supplemental Table 1 for values). There was a trend toward increased production of IL- $1 \beta$ and TNF- $\alpha$ in cell from RRMS subjects not taking DMF compared to healthy controls that appeared to be reversed in RRMS patients taking DMF, but none of these differences were statistically significant (Figures $2 \mathrm{~B}$ and $3 \mathrm{~B}$ ).

\section{Discussion}

To our knowledge, this study is the first to examine the inflammatory response of monocytes isolated from RRMS patients that are being treated with DMF. Previous studies of this nature on other immune cell types demonstrated that DMF treatment resulted in a significant decrease in the frequency of B cells expressing IL-6, TNF- $\alpha$ and GM-CSF, and that DMF treatment improves the responsiveness of MS patients' effector $\mathrm{T}$ cells to allogeneic regulatory $\mathrm{T}$ cells in vitro $[14,15]$. In this study, we show that the amount of IL-6 produced by classically stimulated monocytes was significantly greater from RRMS subjects compared to HC. This finding is in accordance with other reports $[16,17]$. Unique to this study is that the significance of this difference is driven by RRMS subjects that are taking DMF, whose activated monocytes produced more IL- 6 than RRMS patients taking other DMDs. This result is unexpected because previously published results indicate that DMF generally decreases production of inflammatory cytokines, and specifically reduces IL-6 in vitro in many cell types $[8,10,14]$.

Despite being traditionally classified as a pro-inflammatory cytokine, IL-6 can induce an anti-inflammatory cytokine profile, enhancing IL-4/IL-10 production, decreasing IL-1 $\beta$ secretion, and polarizing human monocytes into an anti-inflammatory M2 phenotype under certain co-stimulatory conditions [18-20]. In a specific example, IL-6 treatment of IL-4/IL-13 alternatively activated (M2) monocytes caused release of IL-10, suppression of LPS-induced NO production, and inhibited cytokine production by $\mathrm{CD} 4^{+} \mathrm{T}$ cells; interestingly, this study also demonstrated that in the presence of IFN- $\gamma$ (a classical, inflammatory, M1 activator), IL-6 enhanced production of IL- $1 \beta$ and TNF- $\alpha$, which may suggest that the pleiotropic role of IL- 6 is to enhance, rather than direct the phenotype of macrophage polarization [20]. In one study looking at IL-6 expression in human MS lesions, the highest number of macrophages expressing IL- 6 was found within inactive demyelinating lesions. There was an increase in IL-6 positive cells in lesions with oligodendrocyte preservation, while IL- 6 absence correlated with loss of oligodendrocytes [12]. The traditional M1/M2 macrophage polarization model is now recognized as being overly simplistic. Recent work points to as many as nine different varieties based on transcriptomes from differentially activated monocytes, suggesting that it may be more accurate to think of macrophage phenotypes on a spectrum [21,22]. Notably, active MS lesions contain a major subset of macrophages that display an "intermediate" activation status, displaying both M1 and M2 markers, in accordance with the spectrum model of macrophage phenotypes [23]. All these studies, taken together with our finding of increased LPS-stimulated macrophage production of IL-6, suggest that DMF-induced IL-6 has the potential to act in an anti-inflammatory/protective manner on the mixed phenotype cells present in MS lesions. An alternative explanation for our IL-6 data is that DMF has little or no effect on IL-6 production, while other DMDs suppress it, though not to healthy control levels. In this scenario, it is possible that IL- 6 is acting in a traditionally proinflammatory manner, but IL-6 suppression is simply not a part of the MOA of DMF.

In this study, neither the basal values nor the LPS-stimulated production of IL- $1 \beta$ and TNF- $\alpha$ significantly differed between $\mathrm{HC}$ and RRMS subjects on any DMDs, and there was no significant effect of DMF treatment. Though there are some studies that suggest elevation

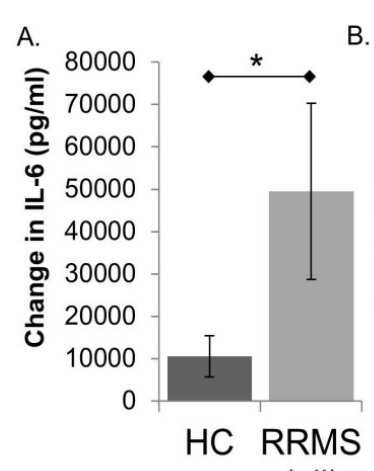

B.

(all)

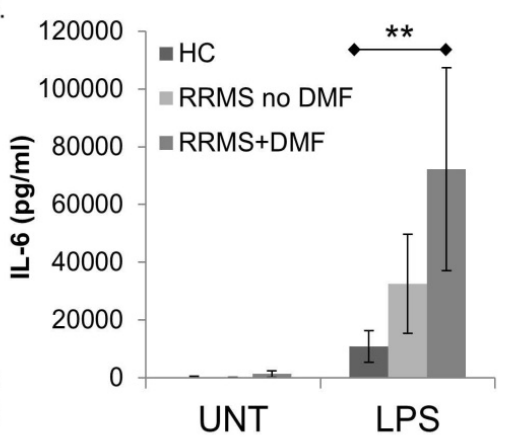

Figure 1. IL-6 production in human monocytes/macrophages from healthy controls (HC) and relapsing-remitting MS (RRMS) subjects. Monocytes/macrophages were enriched by plate adherence from HC or RRMS PBMCs. Cells were not treated (UNT) or treated with LPS $(1 \mu \mathrm{g} / \mathrm{ml})$ for 24 hours prior to collection of supernatants for IL-6 ELISA (eBioscience). In A, bars represent the difference between LPS stimulated values and untreated wells, comparing HC to all RRMS subjects. In B, average UNT and LPS stimulated values are represented separately, and RRMS subjects are divided into those who are taking dimethyl fumarate (RRMS+DMF), and those who are not (RRMS no $\mathrm{DMF})$. Assays were performed in triplicate. * indicates $\mathrm{P}<0.05(0.042)$ comparing HC to RRMS (all). ** indicates $\mathrm{P}<0.05$ (0.017) comparing HC to RRMS+DMF. $\mathrm{N}=19 \mathrm{HC}$ $(16 \mathrm{M} / 3 \mathrm{~F}), 18 \mathrm{RRMS}(16 \mathrm{M} / 2 \mathrm{~F}) ; 10$ RRMS no DMF (9M/1F), 8 RRMS+DMF (7M/1F). Bars are \pm standard error of the mean. 


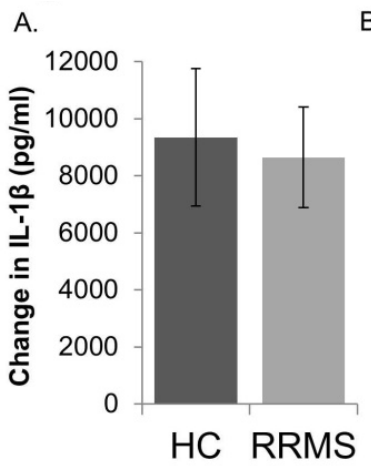

B.

(all)

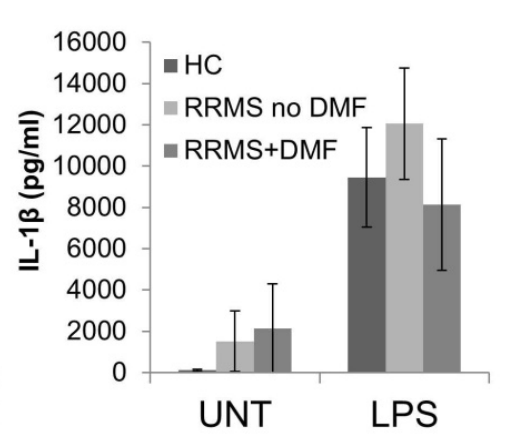

Figure 2. IL-1ß production in human monocytes/macrophages from healthy controls (HC) and relapsing-remitting MS (RRMS) subjects. Monocytes/macrophages were enriched by plate adherence from HC or RRMS PBMCs. Cells were not treated (UNT) or treated with LPS $(1 \mu \mathrm{g} / \mathrm{ml})$ for 24 hours prior to collection of supernatants for IL-1 $\beta$ ELISA (eBioscience). In A, bars represent the difference between LPS stimulated values and untreated wells, comparing HC to all RRMS subjects. In B, average UNT and LPS stimulated values are represented separately, and RRMS subjects are divided into those who are taking dimethyl fumarate (RRMS+DMF), and those who are not (RRMS no DMF). Assays were performed in triplicate. $\mathrm{N}=12 \mathrm{HC}(10 \mathrm{M} / 2 \mathrm{~F}), 12 \mathrm{RRMS}(11 \mathrm{M} / 1 \mathrm{~F}) ; 7$ RRMS no DMF (7M/0F), 5 RRMS+DMF (5M/0F). Bars are \pm standard error of the mean.
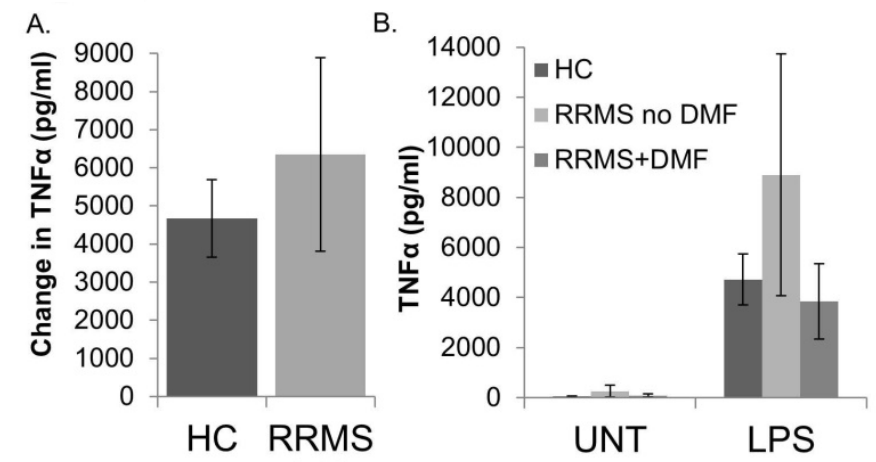

(all)

Figure 3. TNF- $\alpha$ production in human monocytes/macrophages from healthy controls (HC) and relapsing-remitting MS (RRMS) subjects. Monocytes/macrophages were enriched by plate adherence from HC or RRMS PBMCs. Cells were not treated (UNT) or treated with LPS $(1 \mu \mathrm{g} / \mathrm{ml})$ for 24 hours prior to collection of supernatants for TNF- $\alpha$ ELISA (eBioscience). In A, bars represent the difference between LPS stimulated values and untreated wells, comparing HC to all RRMS subjects. In B, average UNT and LPS stimulated values are represented separately, and RRMS subjects are divided into those who are taking dimethyl fumarate (RRMS+DMF), and those who are not (RRMS no DMF). Assays were performed in triplicate. $\mathrm{N}=19 \mathrm{HC}(16 \mathrm{M} / 3 \mathrm{~F}), 17 \mathrm{RRMS}(15 \mathrm{M} / 2 \mathrm{~F}) ; 9 \mathrm{RRMS}$ no DMF $(8 \mathrm{M} / 1 \mathrm{~F}), 8$ RRMS+DMF $(7 \mathrm{M} / 1 \mathrm{~F})$. Bars are \pm standard error of the mean.

of these cytokines in CSF and PBMCs from MS patients, our data are in agreement with others that specifically compared LPS-activated monocytes from healthy control and MS patients [16,17,24,25], indicating that effects on macrophage production of these cytokines may not be an important mechanism of action for these DMDs. A potential confounder of our data is that DMDs may alter the adherence of immune cell populations, potentially decreasing the purity of our monocyte preparations.

Important qualifications for interpretation of the results in this report include the small number of subjects in our RRMS vs. RRMS+DMF subject groups, lack of RRMS subjects who are not taking DMDs, and our high proportion of male subjects. Due to the demographics of those served at the VAPORHCS, our subjects were mostly male; this is unusual for a MS study, given that MS is a disease that is over three times more common in women [26]. There is evidence that men generally have a later age of onset, more rapid disease progression, and a higher prevalence of primary progressive disease $[26,27]$. Some of these sex differences in disease course may be due to differences in immune responses; males have a less robust $\mathrm{T}$ cell response to myelin proteolipid proteins, and less cytokine secretion by peripheral blood lymphocytes in response to stimulation with myelin proteins. Additionally, there is some evidence that males and females respond differently to immunotherapies (IFN- $\beta$ and glatiramer acetate). Also, estrogens influence cytokine and chemokine production, including the cytokines that we examined in this study (IL- $1 \beta$, TNF- $\alpha$, and IL-6) [27,28].

\section{Conclusion}

We consider our finding of elevated IL-6 response in monocytes from RRMS subjects taking DMF an interesting pilot result, which needs to be examined in a larger general population (non-veteran restricted) group. Since there are a greater proportion of women with MS compared to men, it would be of great interest to determine if there are sex-dependent differences in DMF MOA. In addition, our finding that DMD treatment did not result in suppression of IL-6, TNF- $\alpha$ and IL- $1 \beta$ production in RRMS subjects warrants further analysis in a larger cohort. If our results are repeated, new drugs that specifically target monocyte function may prove to be therapeutically effective when used in combination with current DMDs.

\section{Compliance with ethical standards}

The authors declare that they have no conflict of interest.

All procedures performed in studies involving human participants were in accordance with the ethical standards of the institutional and/ or national research committee and with the 1964 Helsinki declaration and its later amendments or comparable ethical standards. Informed consent was obtained from all individual participants included in the study.

\section{Funding}

This work was supported by a Biomedical Laboratory Research and Development Service Merit Review (1I01BX001793), from the United States Department of Veterans Affairs. Oregon Clinical and Translational Institute (OCTRI) provided clinical coordination and nursing services with the support of National Institutes of Health (NIH) grant UL1TR000128 from the National Center for Advancing Translational Sciences (NCATS). The content is solely the responsibility of the authors and does not necessarily represent the official views of the NIH. Department of Veterans Affairs limited subject enrollment to veterans of the US Armed Forces; sponsors had no other role in the study design, in the collection, analysis and interpretation of data, in the writing of the report, nor in the decision to submit the article for publication.

Supplemental Table 1. ELISA results

\begin{tabular}{|c|c|c|c|}
\hline & Healthy Control & RRMS no DMF & RRMS + DMF \\
\hline IL-1 $\beta$ & & & \\
\hline UNT & $106.3 \pm 52.8$ & $1514.4 \pm 1475.4$ & $2142.8 \pm 2140.5$ \\
\hline LPS & $9455.0 \pm 2417.1$ & $12056.4 \pm 2700.5$ & $8131.8 \pm 3192.5$ \\
\hline TNF- $\alpha$ & & & \\
\hline UNT & $48.5 \pm 17.8$ & $259.6 \pm 229.7$ & $85.2 \pm 67.7$ \\
\hline LPS & $4721.0 \pm 1010.0$ & $8904.5 \pm 4838.3$ & $3848.5 \pm 1499.7$ \\
\hline IL-6 & & & \\
\hline UNT & $344.5 \pm 248.9$ & $155.0 \pm 85.2$ & $1279.9 \pm 1115.8$ \\
\hline LPS & $10870.4 \pm 5495.7$ & $32585.8 \pm 17135.4$ & $72186.9 \pm 35156.2$ \\
\hline
\end{tabular}

Values are $\mathrm{pg} / \mathrm{ml}, \pm$ standard error of the mean 


\section{References}

1. Dittel BN (2008) CD4 T cells: Balancing the coming and going of autoimmunemediated inflammation in the CNS. Brain Behav Immun 22: 421-430. [Crossref]

2. Grigoriadis N, van Pesch V (2015) A basic overview of multiple sclerosis immunopathology. Eur J Neurol Suppl 2: 3-13. [Crossref]

3. Engelhardt B, Ransohoff RM (2005) The ins and outs of T-lymphocyte trafficking to the CNS: anatomical sites and molecular mechanisms. Trends Immunol 26: 485-95. [Crossref]

4. Dogan RN, Karpus WJ (2004) Chemokines and chemokine receptors in autoimmune encephalomyelitis as a model for central nervous system inflammatory disease regulation. Front Biosci 9: 1500-1505. [Crossref]

5. Bruck W, Sommermeier N, Bergmann M, Zettl U, Goebel HH, et al. (1996) Macrophages in multiple sclerosis. Immunobiology 195: 588-600. [Crossref]

6. Kuhlmann T, Ludwin S, Prat A, Antel J, Bruck W, et al. (2017) An updated histological classification system for multiple sclerosis lesions. Acta Neuropathol 133: 13-24. [Crossref]

7. Doshi A, Chataway J (2016) Multiple sclerosis, a treatable disease. Clin Med (Lond) 16: s53-s59. [Crossref]

8. Wilms H, Sievers J, Rickert U, Rostami-Yazdi M, Mrowietz U, et al. (2010) Dimethylfumarate inhibits microglial and astrocytic inflammation by suppressing the synthesis of nitric oxide, IL-1beta, TNF-alpha and IL-6 in an in-vitro model of brain inflammation. J Neuroinflammation 7: 30. [Crossref]

9. Lehmann JC, Listopad JJ, Rentzsch CU, Igney FH, von Bonin A, et al. (2007) Dimethylfumarate induces immunosuppression via glutathione depletion and subsequent induction of heme oxygenase 1. J Invest Dermatol 127: 835-845. [Crossref]

10. Miljkovic D, Blazevski J, Petkovic F, Djedovic N, Momcilovic M, et al. (2015) A comparative analysis of multiple sclerosis-relevant anti-inflammatory properties of ethyl pyruvate and dimethyl fumarate. J Immunol 194: 2493-2503. [Crossref]

11. Hofman FM, von Hanwehr RI, Dinarello CA, Mizel SB, Hinton D, et al. (1986) Immunoregulatory molecules and IL 2 receptors identified in multiple sclerosis brain. $J$ Immunol 136: 3239-3245. [Crossref]

12. Schonrock LM, Gawlowski G, Bruck W (2000) Interleukin-6 expression in human multiple sclerosis lesions. Neurosci Lett 294: 45-48. [Crossref]

13. Hofman FM, Hinton DR, Johnson K, Merrill JE (1989) Tumor necrosis factor identified in multiple sclerosis brain. $J$ Exp Med 170: 607-612. [Crossref]

14. Li R, Rezk A, Ghadiri M, Luessi F, Zipp F, et al. (2017) Dimethyl Fumarate Treatment Mediates an Anti-Inflammatory Shift in B Cell Subsets of Patients with Multiple Sclerosis. J Immunol 198: 691-698. [Crossref]
15. Schloder J, Berges C, Luessi F, Jonuleit H (2017) Dimethyl Fumarate Therapy Significantly Improves the Responsiveness of T Cells in Multiple Sclerosis Patients for Immunoregulation by Regulatory T Cells. Int J Mol Sci 18. [Crossref]

16. Chuluundorj D, Harding SA, Abernethy D, La Flamme AC (2014) Expansion and preferential activation of the $\mathrm{CD} 14(+) \mathrm{CD} 16(+)$ monocyte subset during multiple sclerosis. Immunol Cell Biol 92: 509-517. [Crossref]

17. Sarchielli P, Orlacchio A, Vicinanza F, Pelliccioli GP, Tognoloni M, et al. (1997) Cytokine secretion and nitric oxide production by mononuclear cells of patients with multiple sclerosis. J Neuroimmunol 80: 76-86. [Crossref]

18. Frisdal E, Lesnik P, Olivier M, Robillard P, Chapman MJ, et al. (2011) Interleukin-6 protects human macrophages from cellular cholesterol accumulation and attenuates the proinflammatory response. J Biol Chem 286: 30926-30936. [Crossref]

19. Janssens K, Slaets H, Hellings N (2015) Immunomodulatory properties of the IL-6 cytokine family in multiple sclerosis. Ann N Y Acad Sci 1351: 52-60. [Crossref]

20. Fernando MR, Reyes JL, Iannuzzi J, Leung G, McKay DM (2014) The proinflammatory cytokine, interleukin-6, enhances the polarization of alternatively activated macrophages. PLoS One 9: e94188. [Crossref]

21. Xue J, Schmidt SV, Sander J, Draffehn A, Krebs W, Quester I, et al. (2014) Transcriptome-based network analysis reveals a spectrum model of human macrophage activation. Immunity 40: 274-88. [Crossref]

22. Mishra MK, Yong VW (2016) Myeloid cells - targets of medication in multiple sclerosis. Nat Rev Neurol 12: 539-551. [Crossref]

23. Vogel DY, Vereyken EJ, Glim JE, Heijnen PD, Moeton M, et al. (2013) Macrophages in inflammatory multiple sclerosis lesions have an intermediate activation status. $J$ Neuroinflammation 10: 35. [Crossref]

24. Farrokhi M, Etemadifar M, Jafary Alavi MS, Zarkesh-Esfahani SH, Behjati M, et al (2015) TNF-alpha Production by Peripheral Blood Monocytes in Multiple Sclerosis Patients and Healthy Controls. Immunol Invest 44: 590-601. [Crossref]

25. Rudick RA, Ransohoff RM (1992) Cytokine secretion by multiple sclerosis monocytes Relationship to disease activity. Arch Neurol 49: 265-270. [Crossref]

26. Harbo HF, Gold R, Tintore M (2013) Sex and gender issues in multiple sclerosis. Ther Adv Neurol Disord 6:237-248. [Crossref]

27. Greer JM, McCombe PA (2011) Role of gender in multiple sclerosis: clinical effects and potential molecular mechanisms. J Neuroimmunol 234: 7-18. [Crossref]

28. Pfeilschifter J, Koditz R, Pfohl M, Schatz H (2002) Changes in proinflammatory cytokine activity after menopause. Endocr Rev 23: 90-119. [Crossref]

Copyright: $@ 2017$ Fiedler SE. This is an open-access article distributed under the terms of the Creative Commons Attribution License, which permits unrestricted use, distribution, and reproduction in any medium, provided the original author and source are credited. 